\title{
Analytical and numerical solutions for the time and space-symmetric fractional diffusion equation
}

\author{
Qianqian Yang ${ }^{1} \quad$ Ian Turner ${ }^{2} \quad$ Fawang Liu $^{3}$
}

(Received 7 August 2008; revised 18 December 2008)

\begin{abstract}
We consider a time and space-symmetric fractional diffusion equation (TSS-FDE) under homogeneous Dirichlet conditions and homogeneous Neumann conditions. The TSS-FDE is obtained from the standard diffusion equation by replacing the first-order time derivative by a Caputo fractional derivative, and the second order space derivative by a symmetric fractional derivative. First, a method of separating variables expresses the analytical solution of the TSS-FDE in terms of the Mittag-Leffler function. Second, we propose two numerical methods to approximate the Caputo time fractional derivative: the finite difference method; and the Laplace transform method. The symmetric space fractional derivative is approximated using the matrix transform method. Finally, numerical results demonstrate the effectiveness of the numerical methods and to confirm the theoretical claims.
\end{abstract}

http://anziamj . austms.org.au/ojs/index.php/ANZIAMJ/article/view/1400 gives this article, (C) Austral. Mathematical Soc. 2009. Published February 23, 2009. ISSN 1446-8735. (Print two pages per sheet of paper.) 


\section{Contents}

1 Introduction

C801

2 Analytical solutions

C803

3 Numerical methods

C805

3.1 Finite difference method with matrix transform method . . C806

3.2 Laplace transform method with matrix transform method. C808

4 Numerical examples

C809

5 Conclusions

C812

References

C812

\section{Introduction}

A growing number of works in science and engineering deal with dynamical systems described by fractional order equations that involve derivatives and integrals of non-integer order $[1,3,6,16]$. These new models are more adequate than the previously used integer order models, because fractional order derivatives and integrals describe the memory and hereditary properties of different substances [12]. This is the most significant advantage of the fractional order models in comparison with integer order models, in which such effects are neglected. In the context of flow in porous media, fractional space derivatives model large motions through highly conductive layers or fractures, while fractional time derivatives describe particles that remain motionless for extended periods of time [7].

In this article, we consider the following time and space-symmetric fractional diffusion equation (TSS-FDE):

$$
{ }_{t} D_{*}^{\alpha} u(x, t)=-K_{\beta}(-\Delta)^{\beta / 2} u(x, t), \quad 0 \leq t \leq T, \quad 0 \leq x \leq L,
$$


subject to either homogeneous Dirichlet boundary conditions, or homogeneous Neumann boundary conditions

$$
u(0, t)=u(L, t)=0, \quad \text { or } \quad \frac{\partial u}{\partial x}(0, t)=\frac{\partial u}{\partial x}(L, t)=0,
$$

and the initial condition

$$
u(x, 0)=g(x),
$$

where $u(x, t)$ is a solute concentration field and $K_{\beta}$ represents the dispersion coefficient. The operator ${ }_{t} D_{*}^{\alpha}$ is the Caputo time fractional derivative of order $\alpha(0<\alpha<1)$, with starting point at $t=0$, defined as [12]

$$
{ }_{t} D_{*}^{\alpha} u(x, t)=\frac{1}{\Gamma(1-\alpha)} \int_{0}^{t} \frac{\partial u(x, \eta)}{\partial \eta} \frac{d \eta}{(t-\eta)^{\alpha}} .
$$

The symmetric space fractional derivative $-(-\Delta)^{\beta / 2}$ of order $\beta(1<\beta \leq 2)$ is defined by Gorenflo and Mainardi [2], where $\Delta$ is the Laplacian operator.

Physical considerations of a fractional diffusion equation restrict $0<\alpha<$ 1 and $1<\beta \leq 2$, and we assume $K_{\beta}>0$ so that the flow is from left to right. The physical meaning of using homogeneous Dirichlet boundary conditions is that the boundary is set far enough away from an evolving plume such that no significant concentrations reach that boundary $[9,10]$. Also, by assuming homogeneous Dirichlet boundary conditions, we derive that the Riesz fractional derivative is equivalent to the fractional power of Laplacian operator [14], that is, $\partial^{\beta} u(x, t) / \partial|x|^{\beta}=-(-\Delta)^{\beta / 2} u(x, t)$. The physical meaning of using homogeneous Neumann boundary conditions is that the tracer moves freely through the boundaries [11].

In the case of $\alpha=1$ and $\beta=2$, the TSS-FDE (1) reduces to the classical diffusion equation. For $0<\alpha<1$ and $\beta=2$, the TSS-FDE (1) models subdiffusion due to particles having heavy tailed resting times, whereas for $\alpha=1$ and $1<\beta<2$ the TSS-FDE (1) corresponds to the Lévy process [16]. 
Hence, the solution of TSS-FDE (1) is important for describing the competition between these two anomalous diffusion processes. The TSS-FDE was first introduced by Zaslavsky [15] to model Hamiltonian chaos. More recently, an important application of TSS-FDE arose in finance [8], where coupled continuous time random walk (CTRW) models were used to describe the movement of log-prices. In these coupled CTRW models, the probability density functions for the limiting stochastic process solve TSS-FDE.

\section{Analytical solutions}

In this section, using the method of separation of variables, the analytical solution of TSS-FDE (1)-(3) is first derived under homogeneous Dirichlet boundary conditions. For homogeneous Neumann conditions, a similar method can be used to derive the analytical solution.

Setting $\mathfrak{u}(x, t)=X(x) T(t)$ and substituting into (1) yields

$$
{ }_{t} D_{*}^{\alpha} X(x) T(t)+K_{\beta}(-\Delta)^{\beta / 2} X(x) T(t)=0 .
$$

Letting $-\omega$ be the separation constant we obtain two fractional ordinary linear differential equations for $\mathrm{X}(\mathrm{x})$ and $\mathrm{T}(\mathrm{t})$, respectively as

$$
\begin{aligned}
& (-\Delta)^{\beta / 2} X(x)-\omega X(x)=0, \\
& { }_{t} D_{*}^{\alpha} T(t)+K_{\beta} \omega T(t)=0 .
\end{aligned}
$$

Following Ilić et al.'s [4] definition of the fractional Laplacian $(-\Delta)^{\beta / 2}$ defined on a bounded region, (5) is expressed as

$$
\sum_{n=1}^{\infty} c_{n}\left(\lambda_{n}^{2}\right)^{\beta / 2} x_{n}+\omega_{n} \sum_{n=1}^{\infty} c_{n} x_{n}=0 .
$$

Hence, under homogeneous Dirichlet conditions, the eigenvalues of (5) are $\omega_{n}=\lambda_{n}^{\beta}=(n \pi / L)^{\beta}$ for $n=1,2, \ldots$, and the corresponding eigenfunctions 
are nonzero constant multiples of $x_{n}=\sin (n \pi x / L)$. For homogeneous Neumann conditions, the eigenvalues are $\omega_{n}=\lambda_{n}^{\beta}=(n \pi / L)^{\beta}$ for $n=0,1,2, \ldots$, and the corresponding eigenfunctions are $x_{n}=\cos (n \pi x / L)$.

Next, we seek a solution of the TSS-FDE (1) under homogeneous Dirichlet conditions in the form

$$
u(x, t)=\sum_{n=1}^{\infty} T_{n}(t) \sin \left(\frac{n \pi x}{L}\right) .
$$

Substituting (8) into (1) yields

$$
{ }_{t} D_{*}^{\alpha} T_{n}(t)=-K_{\beta} \omega_{n} T_{n}(t) .
$$

Since $\mathfrak{u}(\boldsymbol{x}, \mathbf{t})$ must also satisfy the initial conditions (3)

$$
\sum_{n=1}^{\infty} T_{n}(0) \sin \left(\frac{n \pi x}{L}\right)=g(x), \quad 0 \leq x \leq L,
$$

and therefore

$$
T_{n}(0)=\frac{2}{L} \int_{0}^{L} g(x) \sin \left(\frac{n \pi x}{L}\right) d x, \quad n=1,2, \ldots .
$$

For each value of $\boldsymbol{n},(9)$ and (11) compose a fractional initial value problem. Applying the Laplace transform to (9), we obtain

$$
\widetilde{T}_{n}(s)=\frac{s^{\alpha-1} T_{n}(0)}{s^{\alpha}+K_{\beta} \omega_{n}} .
$$

By using the known inverse Laplace transform [13]

$$
E_{\alpha}\left(-\omega t^{\alpha}\right)=\mathcal{L}^{-1}\left\{\frac{s^{\alpha-1}}{s^{\alpha}+\omega}\right\}, \quad \mathfrak{R}(s)>|\omega|^{1 / \alpha},
$$


we obtain the analytical solution of TSS-FDE (1) under homogeneous Dirichlet conditions as

$$
u(x, t)=\sum_{n=1}^{\infty} T_{n}(t) \sin \left(\frac{n \pi x}{L}\right)=\sum_{n=1}^{\infty} E_{\alpha}\left(-K_{\beta} \omega_{n} t^{\alpha}\right) T_{n}(0) \sin \left(\frac{n \pi x}{L}\right),
$$

where $T_{n}(0)$ is given in (11), and $E_{\alpha}(z)$ is the Mittag-Leffler function [12]

$$
\mathrm{E}_{\alpha}(z)=\sum_{n=0}^{\infty} \frac{z^{\mathrm{n}}}{\Gamma(\mathrm{an}+1)}, \quad \alpha>0 .
$$

Here, when $\alpha=1$, the solution (14) corresponds precisely with the results derived for the Riesz space fractional diffusion equation [14].

Similarly, the analytical solution of TSS-FDE (1) under homogeneous Neumann conditions is

$$
\begin{aligned}
u(x, t) & =\sum_{n=0}^{\infty} T_{n}(t) \cos \left(\frac{n \pi x}{L}\right) \\
& =\frac{1}{2} T_{0}(0)+\sum_{n=1}^{\infty} E_{\alpha}\left(-K_{\beta} \omega_{n} t^{\alpha}\right) T_{n}(0) \cos \left(\frac{n \pi x}{L}\right),
\end{aligned}
$$

where $T_{n}(0)=(2 / L) \int_{0}^{L} g(x) \cos (n \pi x / L) d x, n=0,1,2, \ldots$, and we have used the result that $\mathrm{E}_{\alpha}(0)=1$.

\section{$3 \quad$ Numerical methods}

In this section, we present two numerical schemes to simulate the solution behaviour of TSS-FDE (1)-(3). In Section 3.1, a finite difference method (FDM) and the matrix transform method (MTM) are used to discretize the Caputo time fractional derivative and the symmetric space fractional derivative, respectively. In Section 3.2, using the Laplace transform method (LTM) 
together with the MTM, we transfer the TSS-FDE (1) into a discrete system describing the evolution of $\mathfrak{u}(x, t)$ in space and time.

Using time stepping methods in the fractional case requires the storage of all previous time steps. The difficulty in solving fractional differential equations, particularly where the application area requires a solution to be given over a long time interval, is essentially because fractional derivatives are non-local operators. The so-called non-local property means that the next state of a system not only depends on its current state, but also on the historical states starting from the initial time. This property is closer to reality and is the main reason why fractional Calculus has become more and more useful. To overcome this difficulty, some researchers explore techniques for reducing computational cost that keeps the error under control. The simplest approach is to disregard the tail of the integral and to integrate only over a fixed period of recent history. This is commonly referred to as the 'short-memory' principle, and is described by Podlubny [12]. Here, we only consider the full memory case.

\subsection{Finite difference method with matrix transform method}

Let $x_{l}:=l h, l=0,1, \ldots, M$, where $h:=L / M$ is the space step; $t_{n}:=$ $n \tau, n=0,1, \ldots, N$, where $\tau:=T / N$ is the time step; and $u_{l}^{n}$ denote the numerical approximation of $\mathfrak{u}\left(\mathrm{x}_{\mathrm{l}}, \mathrm{t}_{\mathfrak{n}}\right)$. Adopting the FDM given by Lin \& $\mathrm{Xu}[5]$, we discretize the Caputo time fractional derivative as

$$
{ }_{t} D_{*}^{\alpha} u_{l}^{n+1}=\frac{\tau^{-\alpha}}{\Gamma(2-\alpha)} \sum_{j=0}^{n} b_{j}\left[u_{l}^{n+1-j}-u_{l}^{n-j}\right]+O\left(\tau^{2-\alpha}\right),
$$

written in matrix form

$$
{ }_{\mathrm{t}} \mathrm{D}_{*}^{\alpha} \mathbf{U}^{n+1}=\frac{1}{\mu_{0}} \sum_{j=0}^{n} b_{j}\left[\mathbf{U}^{n+1-j}-\mathbf{U}^{n-j}\right]+\mathbf{O}\left(\tau^{2-\alpha}\right),
$$


where $\mu_{0}=\tau^{\alpha} \Gamma(2-\alpha), b_{j}=(j+1)^{1-\alpha}-j^{1-\alpha}, j=0,1,2, \ldots, n$. Utilising the theory described by Ilić et al. [4], we find a matrix representative for the fractional Laplacian operator

$$
-(-\Delta)^{\beta / 2} \mathbf{U} \approx-\frac{1}{h^{\beta}} \mathbf{A}^{\beta / 2} \mathbf{U},
$$

where $\mathbf{A}=\operatorname{tridiag}(-1,2,-1) \in \mathbb{R}^{(M-1) \times(M-1)}$ under homogeneous Dirichlet conditions, or

$$
\mathbf{A}=\left[\begin{array}{rrrrr}
1 & -1 & & & \\
-1 & 2 & -1 & & \\
& \ddots & \ddots & \ddots & \\
& & -1 & 2 & -1 \\
& & & -1 & 1
\end{array}\right]_{(M+1) \times(M+1)}
$$

under homogeneous Neumann conditions. Since the matrix A is symmetric positive definite (SPD), there exits a nonsingular matrix $\mathbf{P}$ that orthogonally diagonalises $\mathbf{A}$ as

$$
\mathbf{A}=\mathbf{P} \wedge \mathbf{P}^{\top}
$$

where $\Lambda=\operatorname{diag}\left(\lambda_{1}, \lambda_{2}, \ldots, \lambda_{M-1}\right)$ under homogeneous Dirichlet conditions or $\Lambda=\operatorname{diag}\left(\lambda_{0}, \lambda_{1}, \ldots, \lambda_{M}\right)$ under homogeneous Neumann conditions, $\lambda_{i}(i=$ $0,1,2, \ldots, M)$ being the eigenvalues of $\mathbf{A}$. Thus, the fractional Laplacian can be expressed in terms of its spectral decomposition as

$$
-(-\Delta)^{\beta / 2} \mathbf{U} \approx-\frac{1}{h^{\beta}} \mathbf{P} \Lambda^{\beta / 2} \mathbf{P}^{\top} \mathbf{U} .
$$

Now, combining (18) with (21), we obtain the following numerical difference approximation of the TSS-FDE (1):

$$
\frac{1}{\mu_{0}} \sum_{j=0}^{n} b_{j}\left[\mathbf{U}^{n+1-j}-\mathbf{U}^{n-j}\right]=-\eta_{\beta} \mathbf{P} \wedge^{\beta / 2} \mathbf{P}^{\top} \mathbf{U}^{n+1},
$$


where $\eta_{\beta}=K_{\beta} / h^{\beta}$. After simplification,

$$
\left[b_{0} \mathbf{I}+\mu_{0} \eta_{\beta} \mathbf{P} \Lambda^{\beta / 2} \mathbf{P}^{\top}\right] \mathbf{U}^{n+1}=\sum_{j=0}^{n-1}\left(b_{j}-b_{j+1}\right) \mathbf{U}^{n-j}+b_{n} \mathbf{U}^{0},
$$

where $\mathbf{U}^{0}$ is the matrix representation of the initial value $g(x)$.

\subsection{Laplace transform method with matrix transform method}

We now consider an alternate strategy for approximating the fractional ODE system associated with the TSS-FDE (1), when the approximation for the fractional Laplacian is given by (19):

$$
{ }_{t} D_{*}^{\alpha} \mathbf{U}^{n}=-\eta_{\beta} \mathbf{A}^{\beta / 2} \mathbf{U}^{n} .
$$

Applying the Laplace transform to (24) with $\widetilde{\mathbf{U}}^{\mathfrak{n}}=\mathcal{L}\left\{\mathbf{U}^{\mathfrak{n}}(\mathbf{t})\right\}$ yields

$$
\widetilde{\mathbf{U}}^{n}=\left[s \mathbf{I}+s^{1-\alpha} \eta_{\beta} \mathbf{A}^{\beta / 2}\right]^{-1} \mathbf{U}^{0} .
$$

Since A is SPD and has the orthogonal diagonalisation (20), we obtain

$$
\mathbf{U}^{n}=\mathbf{P} \mathcal{L}^{-1}\left\{\left(s \mathbf{I}+s^{1-\alpha} \eta_{\beta} \Lambda^{\beta / 2}\right)^{-1}\right\} \mathbf{P}^{\mathrm{T}} \mathbf{U}^{0} .
$$

Recalling (13) and applying the inverse Laplace transform for each of the eigenvalues, we obtain the second numerical scheme for approximating the TSS-FDE (1) as

$$
\mathbf{U}^{\mathfrak{n}}=\mathbf{P} E_{\alpha}\left(-t_{n}^{\alpha} \eta_{\beta} \Lambda^{\beta / 2}\right) \mathbf{P}^{\top} \mathbf{U}^{0},
$$

where $E_{\alpha}(z)$ is the Mittag-Leffler function defined in (15). 


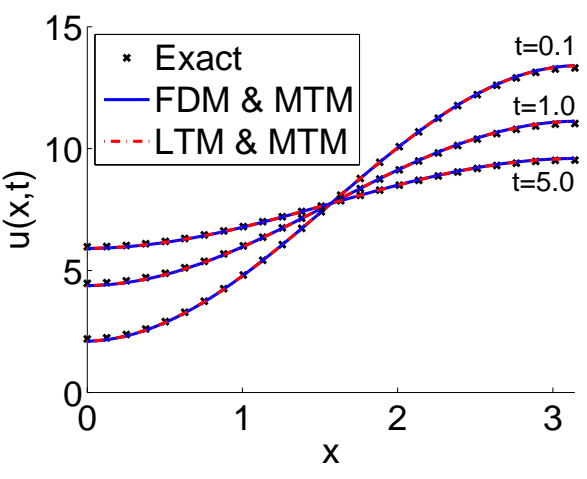

(a)

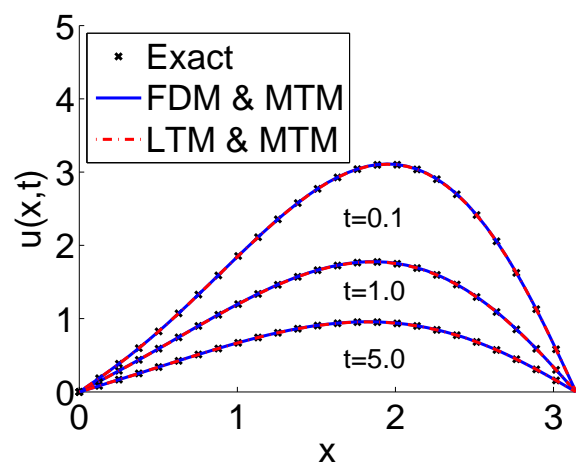

(b)

Figure 1: Comparison of the numerical solutions with the exact solution at different times $t=0.1,1.0,5.0$ for (a) the TSS-FDE (28)-(30), and (b) the TSS-FDE (32)-(34), with $\alpha=0.5, \beta=1.5, M=50, N=100$.

\section{Numerical examples}

In this section, we provide two examples of the TSS-FDE to assess the accuracy of the two numerical schemes proposed in Section 3, and to illustrate the solution behaviour that arises as we change from integer to fractional order in time and space.

Example 1 Considering the following TSS-FDE with homogeneous Neumann boundary conditions:

$$
\begin{aligned}
& { }_{t} D_{*}^{\alpha} u(x, t)=-(-\Delta)^{\beta / 2} u(x, t), \quad 0 \leq t \leq T, \quad 0 \leq x \leq \pi, \\
& \frac{\partial u}{\partial x}(0, t)=\frac{\partial u}{\partial x}(L, t)=0, \\
& u(x, 0)=x^{2}\left(\frac{3}{2} \pi-x\right) .
\end{aligned}
$$


TABLE 1: Maximum errors at $t=1$ with fixed time step $\tau=0.01$.

\begin{tabular}{ccc}
$\mathrm{h}$ & LTM-MTM & FDM-MTM \\
\hline$\pi / 8$ & $1.73 \mathrm{E}-02$ & $1.98 \mathrm{E}-02$ \\
$\pi / 16$ & $4.33 \mathrm{E}-03$ & $7.05 \mathrm{E}-03$ \\
$\pi / 32$ & $1.08 \mathrm{E}-03$ & $4.00 \mathrm{E}-03$ \\
$\pi / 64$ & $2.72 \mathrm{E}-04$ & $3.28 \mathrm{E}-03$ \\
\hline
\end{tabular}

TABLe 2: Maximum errors at $t=1$ with fixed space step $h=\pi / 50$.

\begin{tabular}{ccc}
$\tau$ & LTM-MTM & FDM-MTM \\
\hline $1 / 10$ & $4.44 \mathrm{E}-04$ & $3.43 \mathrm{E}-02$ \\
$1 / 25$ & $4.44 \mathrm{E}-04$ & $1.31 \mathrm{E}-02$ \\
$1 / 50$ & $4.44 \mathrm{E}-04$ & $6.60 \mathrm{E}-03$ \\
$1 / 100$ & $4.44 \mathrm{E}-04$ & $3.44 \mathrm{E}-03$ \\
\hline
\end{tabular}

Following the solution method (16) derived in Section 2, the analytical solution of TSS-FDE (28)-(30) is

$$
u(x, t)=\frac{\pi^{3}}{4}+\sum_{n=1}^{\infty} \frac{12\left[(-1)^{n}-1\right]}{\pi n^{4}} E_{\alpha}\left(-n^{\beta} t^{\alpha}\right) \cos (n x) .
$$

Figure 1(a) shows that both numerical solution schemes provide a good match with the analytical solution (31) at different times $t$, with $\alpha=0.5, \beta=1.5$, $M=50, N=100$.

Example 2 Consider the following TSS-FDE with homogeneous Dirichlet boundary conditions:

$$
\begin{aligned}
& { }_{t} D_{*}^{\alpha} u(x, t)=-(-\Delta)^{\beta / 2} u(x, t), \quad 0 \leq t \leq T, \quad 0 \leq x \leq \pi, \\
& u(0, t)=u(\pi, t)=0 \\
& u(x, 0)=x^{2}(\pi-x)
\end{aligned}
$$




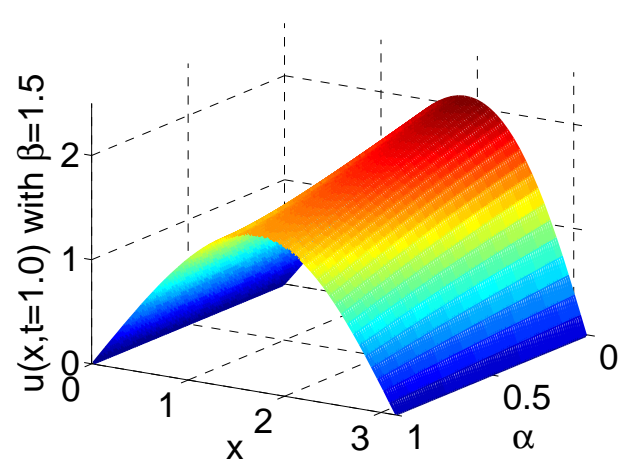

(a)

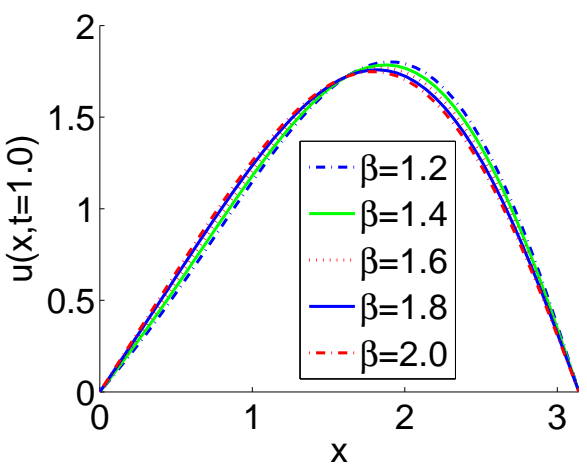

(b)

Figure 2: Numerical solutions at $t=1.0$ for the TSS-FDE (32)-(34) with: (a) $0<\alpha<1, \beta=1.5$; and (b) $\alpha=0.5,1<\beta \leq 2$ (b).

According to (14), the exact solution of TSS-FDE (32)-(34) is

$$
u(x, t)=\sum_{n=1}^{\infty} \frac{8(-1)^{n+1}-4}{n^{3}} E_{\alpha}\left(-n^{\beta} t^{\alpha}\right) \sin (n x) .
$$

Figure 1(b) shows that both numerical solution schemes provide a good match with the analytical solution (35) at different times $t$, with $\alpha=0.5$, $\beta=1.5, M=50, N=100$. Furthermore, Tables 1 and 2 , show that the maximum errors are decreasing as the spatial and temporal nodes increase. Especially, the LTM-MTM is more accurate than the FDM-MTM because it is exact in time. The error observed in the tables for the LTM-MTM is only associated with the spatial discretisation error. More rigorous analyses on stability and convergence will be investigated in future work.

Figure 2(a) displays the solution profiles of the TSS-FDE (32)-(34) over space for $0<\alpha<1, \beta=1.5$ at $t=1.0$. As $\alpha$ is increased over the interval $(0,1)$ the solution profile diminishes in magnitude and becomes slightly more skewed. The solution profiles for selected values of $\beta$ with $\alpha=0.5$ at $t=$ 1.0 are shown in Figure 2(b). The process featured with $\beta=1.2,1.4,1.6,1.8$ 
is slightly more skewed to the right than that with $\beta=2.0$. Furthermore, the solution continuously depends on the time and space fractional derivatives.

\section{Conclusions}

An analytical solution and two numerical schemes for approximating the TSSFDE were derived under both homogeneous Dirichlet and Neumann boundary conditions. These solution techniques can be applied to other fractional partial differential equations. In future research, we will report the stability and convergence analyses of the proposed numerical methods.

Acknowledgements We thank the referees for their thorough reading of the article and the many constructive comments and suggestions. This research has been supported by a $\mathrm{PhD}$ Fee Waiver Scholarship and a School of Mathematical Sciences Scholarship, QUT.

\section{References}

[1] D.A. Benson, S.W. Wheatcraft and M.M. Meerschaert. Application of a fractional advection-dispersion equation. Water Resources Research, 36(6):1403-1412, 2000. doi:10.1029/2000WR900031 C801

[2] R. Gorenflo and F. Mainardi. Random walk models for space-fractional diffusion processes. Fractional Calculus and Applied Analysis, 1(2):167-191, 1998. http://www.ge.infm.it/ecph/papers/ mainardipapers/mainardipap.html. C802

[3] R. Hilfer. Applications of Fractional Calculus in Physics. World Scientific, Singapore, 2000. C801 
[4] M. Ilić, F. Liu, I. Turner, and V. Anh. Numerical approximation of a fractional-in-space diffusion equation (II) - with nonhomogeneous boundary conditions. Fractional Calculus and Applied Analysis, 9(4):333-349, 2006. C803, C807

[5] Y. Lin and C. Xu. Finite difference/spectral approximations for the time-fractional diffusion equation. Journal of Computational Physics, 225(2):1533-1552, 2007. doi:10.1016/j.jcp.2007.02.001 C806

[6] R. Metzler and J. Klafter. The random walk's guide to anomalous diffusion: A fractional dynamics approach. Physics Reports, 339:1-77, 2000. http://www.tau.ac.il/ klafter1/258.pdf C801

[7] M.M. Meerschaert, D.A. Benson, H.P. Scheffler, and B. Baeumer. Stochastic solution of space-time fractional diffusion equations. Physical Review E, 65:041103, 2002.

http://link.aps.org/abstract/PRE/v65/e041103 C801

[8] M.M. Meerschaert and E. Scalas. Coupled continuous time random walks in finance. Physica A, 370:114-118, 2006.

http://arxiv.org/abs/physics/0608281 C803

[9] M.M. Meerschaert, H.P. Scheffler, and C. Tadjeran. Finite difference methods for two-dimensional fractional dispersion equation. Journal of Computational Physics, 211(1):249-261, 2006. doi:10.1016/j.jcp.2005.05.017 C802

[10] M.M. Meerschaert and C. Tadjeran. Finite difference approximations for two-sided space-fractional partial differential equations. Applied Numerical Mathematics, 56(1):80-90, 2006. doi:10.1016/j.apnum.2005.02.008 C802

[11] M.M. Meerschaert and C. Tadjeran. Finite difference approximations for fractional advection-dispersion flow equations. Journal of Computational and Applied Mathematics,172(1):65-77, 2004 doi:10.1016/j.cam.2004.01.033 C802 
[12] I. Podlubny. Fractional Differential Equations. Academic Press, New York, 1999. C801, C802, C805, C806

[13] S.G. Samko, A.A. Kilbas, and O.I. Marichev. Fractional Integrals and Derivatives: Theory and Applications. Gordon and Breach Science Publishers, Amsterdam, 1993. C804

[14] Q. Yang, F. Liu, and I. Turner. Computationally efficient numerical methods for the fractional partial differential equations with the Riesz space fractional derivatives. Appl. Math. Model., Submitted, 2008. C802, C805

[15] G.M. Zaslavsky. Fractional kinetic equation for Hamiltonian chaos. Physica D: Nonlinear Phenomena, 76:110-122, 1994. C803

[16] G.M. Zaslavsky. Chaos, fractional kinetics, and anomalous transport. Physics Reports, 371(6):461-580, 2002. C801, C802

\section{Author addresses}

1. Qianqian Yang, School of Mathematical Sciences, Queensland University of Technology, GPO Box 2434, Queensland 4001, Australia.

mailto:q.yang@qut.edu.au

2. Ian Turner, School of Mathematical Sciences, Queensland University of Technology, GPO Box 2434, Queensland 4001, Australia. mailto:i.turner@qut.edu.au

3. Fawang Liu, School of Mathematical Sciences, Queensland University of Technology, GPO Box 2434, Queensland 4001, Australia.

mailto:f.liu@qut.edu.au 